Check for updates

Cite this: RSC Adv., 2018, 8, 30404

\title{
2D-BN nanoparticles as a spectroscopic marker and drug delivery system with protection properties $\uparrow$
}

\author{
O. P. Gnatyuk, (D) *a G. I. Dovbeshko, (D) *a A. Yershov, (DD b S. O. Karakhim, ${ }^{\text {c }}$ \\ O. Ilchenko (D) and O. Yu. Posudievsky (D)
}

An application of 2D-BN nanoparticles as a spectroscopic marker, weak luminescent marker and anticancer drug (doxorubicin, DOX) delivery system with protection properties was studied for the LNCaP strains of cancer cells using FTIR and Raman spectroscopy for analysing the cancer cells, cells with BN, the cancer cells with DOX, and the cancer cells with BN nanoparticles loaded by DOX. Study of IR absorption and Raman spectra of the LNCaP strains of cancer cells incubated with 2D-BN nanoparticles for 1 hour showed that the 2D-BN nanoparticles could pass through the cell membrane and localize inside the membrane or close to the membrane in the cytoplasm of the cells. We registered the spectra of the disturbed lipids during the DOX-2D-BN passing through the membrane. After incubation for 2 hours and more, spectral changes in other structural components of the cell (nuclei, cytoplasm, mitochondria) can be registered. Confocal microscopy showed that a gold nanostructured support enhances the fluorescence of the cancer cells with 2D-BN as well as that with DOX, however the double action of 2DBN and DOX on the cancer cells aggravates the emission property of the studied system. An MTT test showed that the toxicity of DOX on the 2D-BN nanoparticles is less than that on the reference cells, and at the same time the efficiency of the DOX action on the cancer cells does not change.

Received 13th June 2018
Accepted 13th August 2018

DOI: $10.1039 / c 8 r a 05085 f$

rsc.li/rsc-advances

introduced into clinical practice. The main obstacle is the high

\section{Introduction}

Despite the significant progress achieved in the treatment of various forms of cancer, the problem of acquired resistance of tumor cells due to the use of chemotherapeutic agents is still relevant, as is the issue of developing drug delivery systems that could improve the efficiency and specificity of therapeutic cancer treatment methods.

The possibility of using nanoparticles to solve these problems has been studied for many years, and the spectrum of the nanoparticles is the most extensive - from metal ( $\mathrm{Au}, \mathrm{Cd}, \mathrm{Fe}$ ) to nonmetallic $\left(\mathrm{Fe}_{2} \mathrm{O}_{3}, \mathrm{SiO}_{2}, \mathrm{TiO}_{2}, \mathrm{Al}_{2} \mathrm{O}_{3}\right)$ and carbon (fullerenes, graphene, nanotubes). ${ }^{1-3}$ However, these numerous studies did not lead to significant progress; many developments still remain at the stage of laboratory research and are not being

${ }^{a}$ Department of Physics of Biological Systems, Institute of Physics of the National Academy of Sciences of Ukraine, Prospekt Nauki 46, Kiev 03039, Ukraine. E-mail: hrysantemka@gmail.com

${ }^{b}$ Institute of Molecular Biology and Genetics of the National Academy of Sciences of Ukraine, Zabolotnogo Str. 150, Kyiv 03680, Ukraine

'Palladin Institute of Biochemistry of the National Academy of Sciences of Ukraine, Leontovicha Street 9, Kyiv 01601, Ukraine

${ }^{d}$ Department of Micro \& Nanotechnology, Technical University of Denmark, Denmark. E-mail: olil@nanotech.dtu.dk

${ }^{e}$ L.V. Pisarzhevsky Institute of Physical Chemistry of the National Academy of Sciences of Ukraine, Prospekt Nauki 31, Kyiv 03028, Ukraine

$\dagger$ Electronic supplementary information (ESI) available. See DOI: 10.1039/c8ra05085f cytotoxicity of nanoparticles, both in relation to tumour cells and normal healthy cells. When penetrating through cell membranes, nanoparticles can form complexes with biological molecules (proteins and DNA, as well as with the cell membrane itself), thus impeding normal functioning of the biomolecules.

Biological nanoparticles (DNA and RNA molecules, viruses, enzymes, ribosomes, vesicles etc.) as well as complexes of organic polymers with medicinal preparations have proven to be better. But there are limitations associated with the possible change in the structure of the medication, and therefore the change in its properties and efficacy in this case.

An active study of graphene has stimulated interest in research of other kinds of $2 \mathrm{D}$ nanomaterials, a unique place among which is possessed by boron nitride (BN). The uniqueness of BN monolayer is that it may have a crystal structure similar to that of graphene, as well as similar physical properties, with the exception of the band gap width - BN is a typical dielectric with a band gap of $\sim 5 \mathrm{eV}$. This is due to the ionic nature of this material. At the same time, its physical properties and intrinsic luminescence have characteristics similar (close) to those of diamond nanoparticles. ${ }^{4}$ The absence of absorption in the visible range could be one of the advantages of this material for biological applications.

For example, hexagonal BN, h-BN, in contrast to the abovementioned nanoparticles, did not exhibit cytotoxicity to a number of cell cultures, as shown in several studies. In 
particular, BN nanoparticles of $100-200 \mathrm{~nm}$ size are not cytotoxic to the cell line of osteoblasts, ${ }^{5}$ while BN nanotubes do not exhibit cytotoxicity to the neuroblastoma and are able to penetrate into the cell via phagocytosis. ${ }^{6,7}$ Also, attempts to use BN nanoparticles for the development of drug delivery systems ${ }^{8}$ and antitumour therapy ${ }^{9,10}$ are described literature..$^{11,12}$

$\mathrm{BN}$ is isoelectronic to carbon and can form a number of allotropic modifications - hexagonal graphite-like and cubic diamond-shaped - according to two types of hybridization - $\mathrm{sp}^{2}$ corresponding to hexagonal and rhombohedral crystal lattices, and $\mathrm{sp}^{3}$ corresponding to cubic and wurtzite-like ones. The hexagonal and cubic type of BN lattice are analogues of graphite and diamond, respectively. During synthesis of 2D-BN nanoparticles by chemical vapor deposition, different allotropic modifications can be formed such as amorphous and cubic BN, additional impurities could be included too. This synthesis procedure itself requires the use of high temperatures, pressures and significant energy costs. ${ }^{13-15}$

In the present paper we propose the use of $2 \mathrm{D}-\mathrm{BN}$ nanoparticles prepared by liquid exfoliation of the preliminary solvent-free mechanochemically delaminated bulk h-BN. Our objective was to study the biophysical properties of the prepared nanoparticles, to test their cytotoxicity and to analyze their effect on the cell components, as well as to define the possibility of their use as fluorescent markers for insertion of nonluminescent antitumour drugs into the cell that would point on the location of 2D-BN as well indicate a place of location of the cell component near the 2D-BN. The aim of the work was also the visualization of the presence of antitumour drug (e.g. doxorubicin) in the cells without additional use of dyes and other fluorescent labels. We used infrared spectroscopy to evaluate molecular interaction of 2D-BN nanoparticles with the cell components (lipids, proteins, DNA etc.) and Raman spectroscopy for visualization of BN nanoparticles inside the cell indicated on the component cell.

\section{Preparation of 2D-BN nanoparticles}

A dry mixture of $50 \mathrm{mg}$ of h-BN (\#10043-11-5, Alfa Aesar) and $2 \mathrm{~g}$ of $\mathrm{NaCl}$ (\#S7653, Aldrich) used as the delamination agent was mechanochemically treated in an argon atmosphere using an agate grinding bowl of a planetary ball mill Pulverisette 6 (Fritsch) at a rotation rate of $500 \mathrm{rpm}$ for $1 \mathrm{~h}$. The weight ratio of reactants to milling media was about $1: 20$. The delamination agent was removed by washing with water, and the obtained product - nanostructured h-BN (nh-BN) - was dried in vacuum at $60^{\circ} \mathrm{C}$. The yield of nh-BN was $40 \mathrm{mg} .{ }^{16}$ The preparation of 2DBN was carried out in water by ultrasonic disintegration (Sonopuls 2070) of nh-BN for $1 \mathrm{~h}$. The resulting dispersions were purified by centrifugation (5430, Eppendorf) at $2000 \mathrm{rpm}$ during $1.5 \mathrm{~h}$. 2D-BN nanoparticles were characterized by a series of mutually complimentary experimental methods and the obtained data are summarized in ESI (Fig. S1-S6†).

\section{Preparation of 2D-BN:DOX}

2D-BN:DOX was prepared by mixing $12.5 \mu \mathrm{L}$ of $2 \mathrm{D}-\mathrm{BN}$ dispersion $\left(0.3 \mathrm{mg} \mathrm{mL}^{-1}\right)$ with $5 \mu \mathrm{L}$ of DOX solution, and $82.5 \mu \mathrm{L}$ of phosphate buffer, subsequent incubation during for 24 hours, centrifugation at $5500 \mathrm{rpm}$ for $90 \mathrm{~min}$ and $10000 \mathrm{rpm}$ for $1 \mathrm{~min}$. The volume of solution was restored by adding $100 \mu \mathrm{L}$ of phosphate buffer. We tested optical absorbance at $\lambda=496 \mathrm{~nm}$ with Nanodrop 2000 (ThermoScientific, USA). The DOX optical absorbance was equal to 0.052 and that of 2D-BN:DOX to 0.019 . For the estimation of cytotoxicity of 2D-BN:DOX (for reference and cancer cells). Comparative spectral characterization of 2DBN and 2D-BN:DOX is shown in Fig. S7 and S8. $\dagger$

\section{Cell treatment with 2D-BN nanoparticles and sample preparation for vibrational spectroscopy}

To study the effect of 2D-BN nanoparticles on living cells, the model line of LNCaP tumour cells was used. They were adherent epithelial cells of adenocarcinoma of human prostate gland, which are sensitive to androgen. It was believed that LNCaP reproduce the early stages of prostate cancer development. ${ }^{17}$ Their approximate size was $10-12 \mu \mathrm{m}$ in an unattached state, and it could be larger when attached to the culture surface. The human prostate adenocarcinoma LNCaP cell line was kindly provided by Dr V. Kashuba, (Karolinska Institute, Stockholm, Sweden).

Two sets of experiments were carried out to analyze the effect of 2D-BN nanoparticles on the LNCaP cells. Within the first one, different times of the cells incubation with the nanoparticles (from one to ten hours) were used to detect time dependency, and the second one was based on one-day incubation. LNCaP cells were grown in a 6-cup plate in amount of $3 \times 10^{5}$ cells per cup (designated as "treated" and "reference" ones), and cultured under standard conditions (DMEM, 10\% FBS, in 5\% $\mathrm{CO}_{2}$ atmosphere). The following day, $2 \mathrm{D}-\mathrm{BN}$ aqueous dispersion was added to the cups (the final concentration in the nutrient medium was $0.3 \mathrm{mg} \mathrm{mL}^{-1}$ ), and after the appropriate incubation time (the final cell number in the cell was $1 \times 10^{6}$ ) the nutrient medium was removed, washed twice with $1 \times$ PBS, and then the cells were disconnected from the culture plastics during 5 min by a trypsin-EDTA mixture.

The cells were harvested by centrifugation in $1.5 \mathrm{~mL}$ test tubes at $13000 \mathrm{rpm}$ for $1 \mathrm{~min}$ followed by removal of supernatant, subsequent double washing with PBS (Sigma-Aldrich) and drying of the precipitate in vacuum. At the end of the cell treatment with 2D-BN, each cup of the tablet contained approximately $1 \times 10^{6}$ cells (3-4 mg).

\section{D-BN cytotoxicity assay and visualization of their in-cell location using dyes}

For the cytotoxicity assay of 2D-BN nanoparticles, NIH3T3 mouse culture fibroblasts (as a model of normal non-tumour cells) were used. The mouse embryonic fibroblast NIH3T3 cell line was kindly provided also by Dr V. Kashuba, (Karolinska Institute, Stockholm, Sweden).

The cells were scattered in a 96-well plate the day before their treatment in the amount of 2000 cells per well. An aqueous solution of 2D-BN was added to achieve a final concentration of $1,2,5,10,20,50$ and $100 \mu \mathrm{g} \mathrm{mL}^{-1}$. The cell viability was measured using the MTT test (Fig. 1) as the percentage of 2D- 


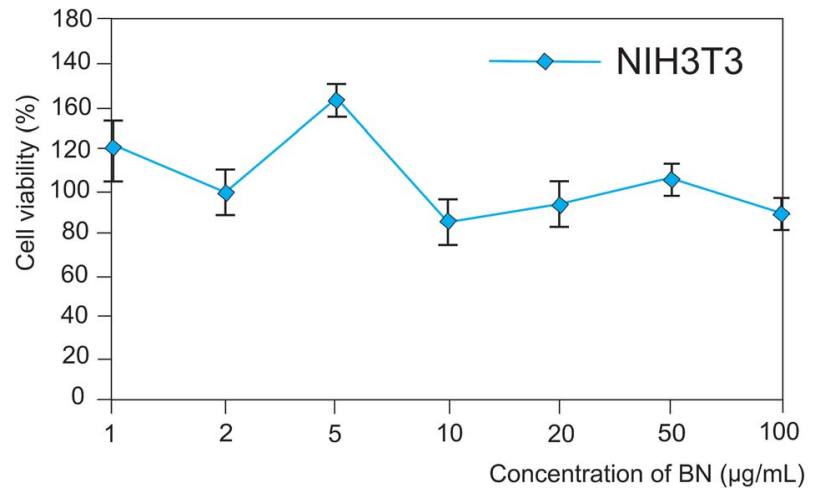

Fig. 1 The vitality of 2D-BN treated NIH3T3 cells.

BN-treated live cells against reference cells. Standard deviations were measured for each three repetitions.

To evaluate the effect of 2D-BN nanoparticles on cytotoxicity of the anticancer drug doxorubicin (Dox), the NIH3T3 (normal) or the LNCaP (tumour) cells were dispersed in a 96-well plates the day before their 2D-BN treatment in amount of 2000 cells per well. The 2D-BN:Dox complex (with optical absorption of 0.019-0.021) and Dox solution (12.5 $\mathrm{mg} \mathrm{mL}^{-1}$, optical absorption 0.020) of equivalent optical absorption were added per $200 \mathrm{~mL}$ of cell medium (DMEM) in the following amounts: 3.2, 8,16 and $32 \mu \mathrm{L}$ for achieving the final Dox concentration of 0.2 , $0.5,1$ and $2 \mathrm{mg} \mathrm{mL}^{-1}$. Cell viability was measured by MTT-test (Fig. 2) as a percentage of live cells treated with complex 2D$\mathrm{BN}$ :Dox or pure Dox against reference cells. Standard deviations were measured for each three repetitions.

As could be seen in Fig. 2, 2D-BN does not inhibit the vitality of NIH3T3 cells, and an increase in its concentration in the nutrient medium up to $100 \mathrm{mg} \mathrm{mL}^{-1}$ did not lead to an increase in the proportion of dead cells compared to the untreated ones. Similar studies carried out on the cell treated with 2D-BN:Dox complex allowed us to found out that addition of 2D-BN:Dox did not change the viability of the NIH3T3 and the LNCaP cells compared to the addition of the similar amounts of pure Dox. This allowed us to conclude that $2 \mathrm{D}-\mathrm{BN}$ is non-toxic to cells and does not affect the toxicity of Dox within the prepared 2DBN:Dox complexes in accord with Fig. S9. $\dagger$

\section{Vibrational spectroscopy investigation of 2D-BN nanoparticles} interaction with LNCaP tumour cells

Raman and FTIR measurements. The FTIR spectra were collected in the $380-5300 \mathrm{~cm}^{-1}$ region with an IFS-66 Bruker instrument. Deconvolution and decomposition of the bands was performed using Opus-4.0 program.

The Raman spectra of cell were obtained by home-made Raman instrument with verification of its ability for RS registration. ${ }^{18}$ In this study was used two lasers (532 and $785 \mathrm{~nm}$ ) with power on sample about $10 \mathrm{~mW} \mu \mathrm{m}^{-2}$ at $532 \mathrm{~nm}$ and 20 $\mathrm{mW} \mu \mathrm{m}^{-2}$ at $785 \mathrm{~nm}$. Dry objective Olympus Plan-Apochromat $40 \times / 0.95$ in inverted microscope configuration was used. Laser was focused on the cells placed directly on the $\mathrm{CaF}_{2}$ surface.

Astigmatism-free Princeton Instruments Schmidt-CzernyTurner spectrograph IsoPlane SCT-320 was used in our Raman system. Spectrograph has focal length $320 \mathrm{~mm}$ with aperture ratio $\mathrm{f} / 4.6$. Measurements were carried out on grating with $600 \mathrm{~g} \mathrm{~mm}^{-1}$ (blaze angle at $860 \mathrm{~nm}$ ), which provide spectral resolution about $5 \mathrm{~cm}^{-1}$ in the ranges $100-3800 \mathrm{~cm}^{-1}$ from laser wavelength $532 \mathrm{~nm}$ and $2.5 \mathrm{~cm}^{-1}$ in the range 200$1900 \mathrm{~cm}^{-1}$ from laser wavelength $785 \mathrm{~nm}$. Spectra were collected with nitrogen cooled CCD PyLoN 400BR eXcelon (Princeton Instruments). Wavelength and spectral sensitivity calibration of the Raman system was done with neon lamp and calibrated $45 \mathrm{~W}$ quartz tungsten halogen lamp (model 63358 from Newport Corp.), respectively. Detailed description of the Raman system setup was described in (ref. 18). Wavelength calibration was done automatically during start of the system software and after every switch of gratings in the spectrograph. Spectral sensitivity calibration was done periodically every month. For the data analysis we used WiRE3.4 software. ${ }^{18}$

Time dependence of $2 \mathrm{D}-\mathrm{BN}$ nanoparticles interaction with LNCaP tumour cells. To study the time dependence of 2D-BN nanoparticles interaction with tumour cells, we have carried out a set of experiments with different times of the cultivation of the tumour cells treated with nanoparticles. The cells were cultivated for 1,2 and $10 \mathrm{~h}$; the reference sample without nanoparticles was cultivated for $2 \mathrm{~h}$ and the corresponding FTIR data are presented in Fig. 3. The most interesting results were obtained for the sample after $1 \mathrm{~h}$ cultivation. As one could see from Fig. 3, upon the 2D-BN treatment, a significant
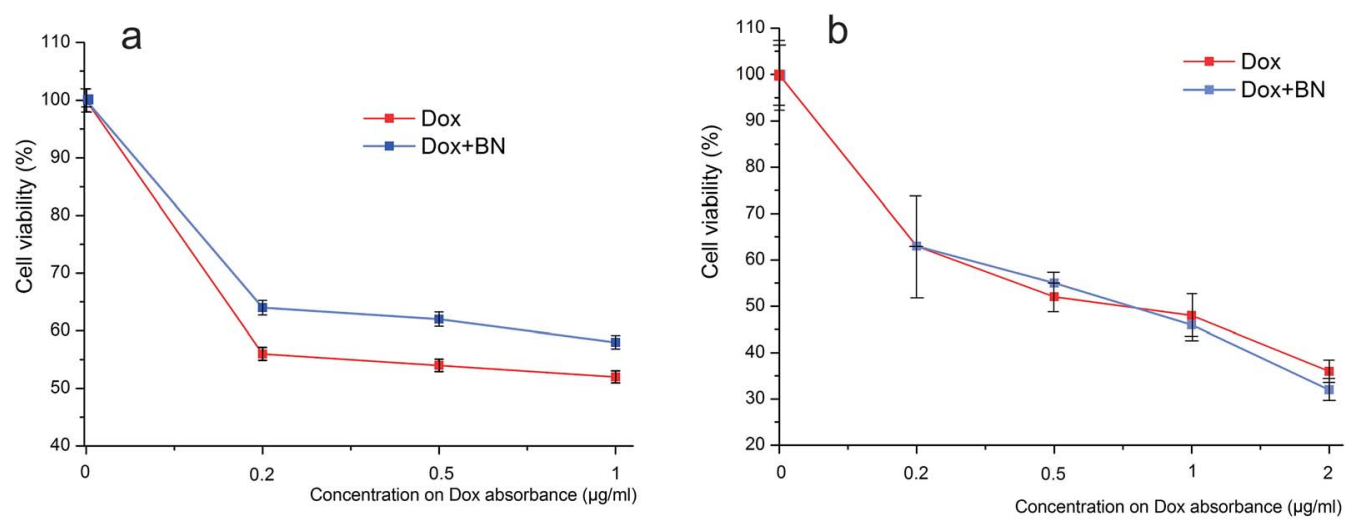

Fig. 2 The vitality of 2D-BN:Dox treated NIH3T3 (a) and LNCaP (b) cells. 


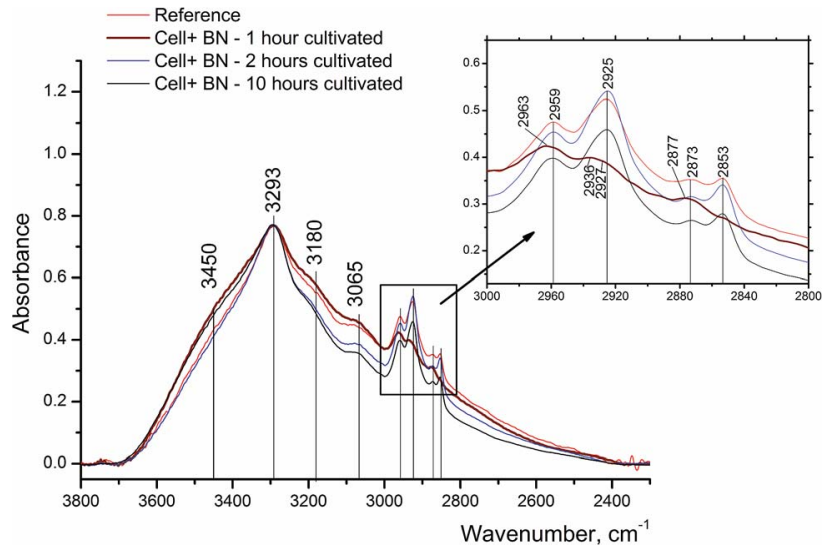

Fig. 3 FTIR absorption spectra of LNCaP cell cultures without (reference) and after treatment with 2D-BN nanoparticles using 1, 2 and $10 \mathrm{~h}$ incubation time in the region of $2400-3600 \mathrm{~cm}^{-1}$.

redistribution in the intensity of phospholipid $\mathrm{CH}_{3}$ (the absorption bands at 2959 and $2871 \mathrm{~cm}^{-1}$ ) and $\mathrm{CH}_{2}$ (the absorption bands at 2927 and $2853 \mathrm{~cm}^{-1}$ ) groups in favour of the first is observed. In the control sample and in the samples after 2 and 10 hours of cultivation the absorbance of $\mathrm{CH}_{2}$ groups was predominating. ${ }^{19-25}$

Such intensity redistribution suggests an effective interaction of 2D-BN nanoparticles with cell membranes, whereby the spectral changes take place at the first hour after the cell treatment. In the FTIR spectrum of 2D-BN:LNCaP cells after $1 \mathrm{~h}$ of cultivation (Fig. 4) some changes in the amide vibration region are observed consisting in the low-frequency shift of Amide I maximum from $1654 \mathrm{~cm}^{-1}$ in the reference $(\alpha$-spiral with signs of disordered form) to $1650 \mathrm{~cm}^{-1}$ ( $\alpha$-spiral proteins), and the shoulder emergence at $1639 \mathrm{~cm}^{-1}$ ( $\beta$-conformation of proteins). ${ }^{19,20}$ The shoulder emergence at about $1120 \mathrm{~cm}^{-1}$, which could be attributed to $\mathrm{C}-\mathrm{O}$ and $\mathrm{P}-\mathrm{O}-\mathrm{C}$ phospholipid vibration, indicates the interaction of the nanoparticles with the cell membrane..$^{21-25}$

Thus, it could be concluded that the 2D-BN nanoparticles penetrate through the membranes into the cells very fast (within 1 hour), thereby altering significantly the cell membrane structure.

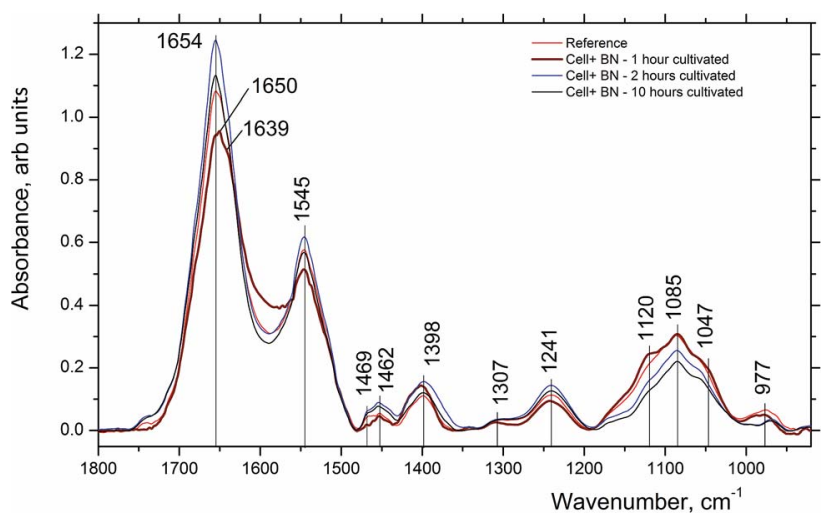

Fig. 4 FTIR absorption spectra of LNCaP cell cultures without (reference) and after treatment with 2D-BN nanoparticles for 1, 2 and $10 \mathrm{~h}$ incubation time in the region of $1000-1800 \mathrm{~cm}^{-1}$.
Study of 2D-BN nanoparticles interaction with LNCaP tumour cells using Raman spectroscopy. After analyzing a number of micro-Raman spectra of the studied cells, we came to the conclusion that the position and intensity of the observed Raman bands essentially depends on the spectrum registration spot, which could be the cell nucleus, cytoplasm, cellular organelles (in particular, mitochondria), cell membranes, etc. When the 2D-BN nanoparticle occurs at the registration spot, the intensity of the Raman bands increased in 2-5 times, and the spectrum as a whole was recorded better. This could be due to reflection of the exciting radiation from the nanoparticle and its subsequent passage through the corresponding cellular organelle. In other words, the $2 \mathrm{D}-\mathrm{BN}$ nanoparticles served as a reflecting mirror that multiply directs the excitatory light onto the cell components. Thus, when the 2D-BN nanoparticle after cell treatment is located on the cell membrane, then the registered Raman spectrum will be a characteristic of the membrane. Therefore it is difficult to reproduce these spectra, since it is difficult to direct the exciting light onto a certain cell organelle.

However, if one choose the right incubation time, it could be possible to estimate where the nanoparticles would be located. For example, the Raman spectrum of the LNCaP cell cultures enhanced by the 2D-BN nanoparticles located near or at the cell membrane is presented in Fig. 5. The sample was a thick drop of 2D-BN-treated LNCaP cells suspension at the time of scanning the cells deep into $Z$ axis. It is seen from the figure that the main contribution to the spectrum is given by lipids and membrane proteins, with large advantage of the bands from the lipid fractions, cholesterol in particular. The Raman bands at $616,1306,1437,1605$ and $1656 \mathrm{~cm}^{-1}$ could be attributed to lipids, and the band at $1001 \mathrm{~cm}^{-1}$ probably refers to free phenyl alanine. ${ }^{26}$

Other Raman spectra, which were reproduced with lesser changes when taken at different points of the sample, looked significantly different as compared to the above mentioned spectrum (Fig. 6). The band at $1657 \mathrm{~cm}^{-1}$ has a complex structure, since besides lipids and proteins, the DNA of the cell nucleus could also contribute to this band. This is evidenced by the appearance of the band at $1338 \mathrm{~cm}^{-1}$ and other bands in its vicinity, assigned to adenine, as well as new band at $780 \mathrm{~cm}^{-1}$.

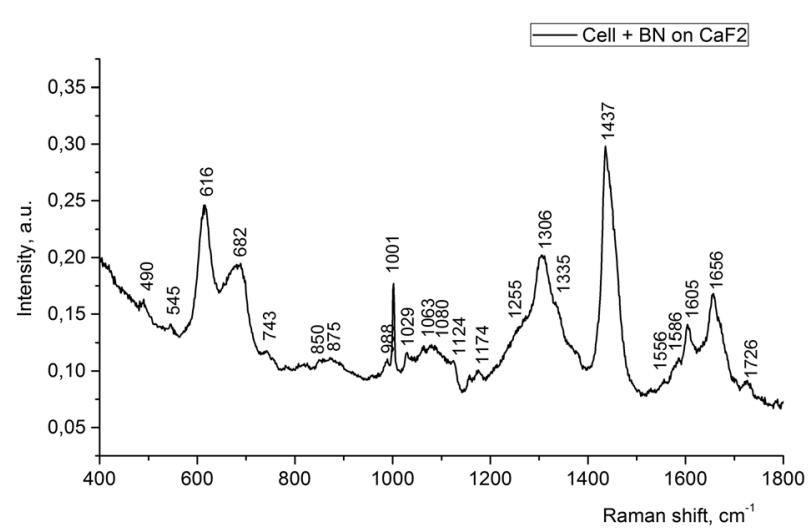

Fig. 5 Raman spectra of LNCaP cell cultures after treatment with 2DBN nanoparticles (1 day incubation time). 


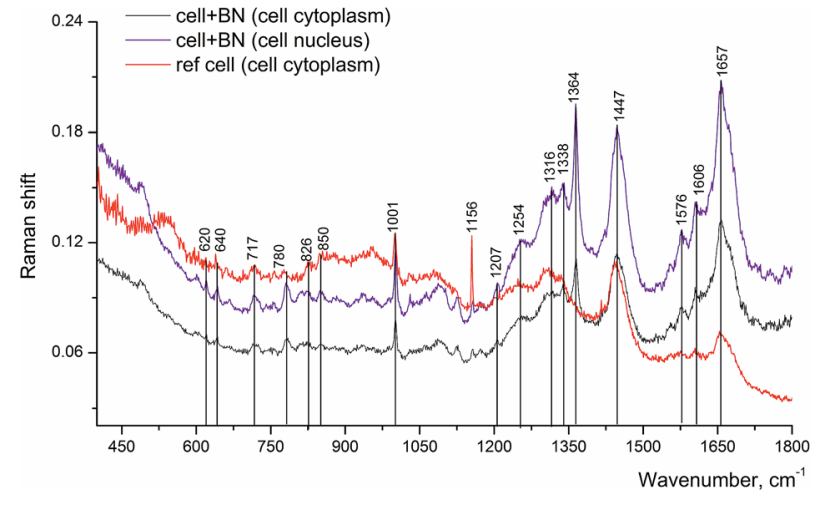

Fig. 6 Raman spectra of LNCaP cell cultures without (reference) and after processing with 2D-BN nanoparticles (1 day incubation time).

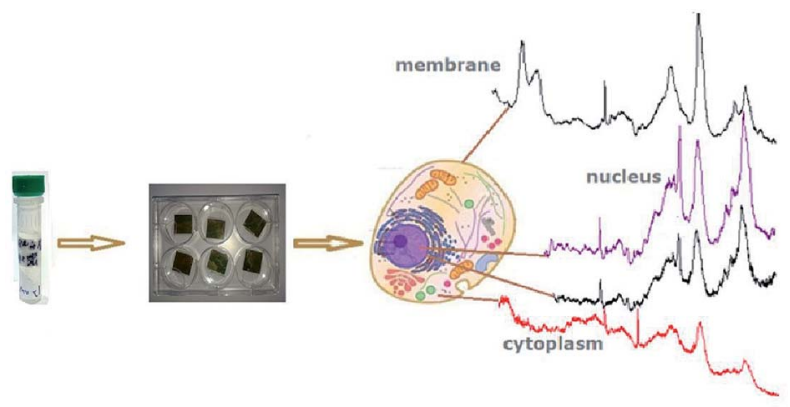

Fig. 7 Schematic representation of the cell cytological analysis using Raman spectroscopy.

Thus, we could assume that the place in the cell, from which we registered this spectrum, is the cell nucleus or the region nearby.

The Raman band at $1001 \mathrm{~cm}^{-1}$ is connected with phenylalanine and it is characteristic of the cell protein observed in the spectrum of any cell. The band at $1657 \mathrm{~cm}^{-1}$ is assigned to Amide I, the band at $1606 \mathrm{~cm}^{-1}$ could be attributed to $\mathrm{C}=\mathrm{C}$ bonds of amino acids, in particular phenylalanine, as well as $\mathrm{NH}_{2}$ groups; the band at $1447 \mathrm{~cm}^{-1}$ is probably due to $\mathrm{CH}_{2}$ deformation vibrations of phospholipids. ${ }^{26}$ With the use of Raman spectroscopy, it was possible to detect the presence of 2D-BN nanoparticles in the cell, as evidenced by characteristic $1364 \mathrm{~cm}^{-1}$ band. In the reference sample (tumour cells without 2D-BN nanoparticles treatment) this band was absent (Fig. 6).

The spectrum of reference cells (without treatment with 2DBN nanoparticles) differs from the spectra of the 2D-BN treated cells by the intensity ratio between the Raman bands (Fig. 7).

Here, the band at $1447 \mathrm{~cm}^{-1}$ (CH deformation vibrations) is the most intense, and the bands at about $1500-1800 \mathrm{~cm}^{-1}$ (characteristic bands of proteins and nucleic acid bases) are weaker, which suggests that the spectrum refers to the cytoplasm. In addition, the spectrum of the reference cells is characterized by a higher level of noise and a poorer separation of the fine structure of the bands.

Thus, using Raman spectroscopy, the spectra from different components of the cell and cellular organelles can be

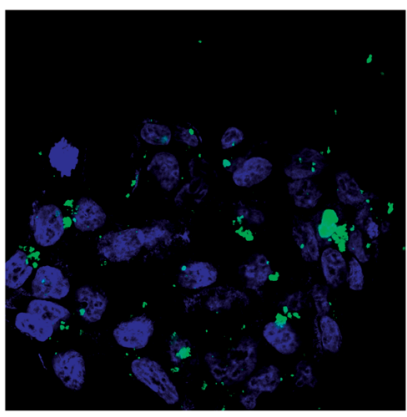

a

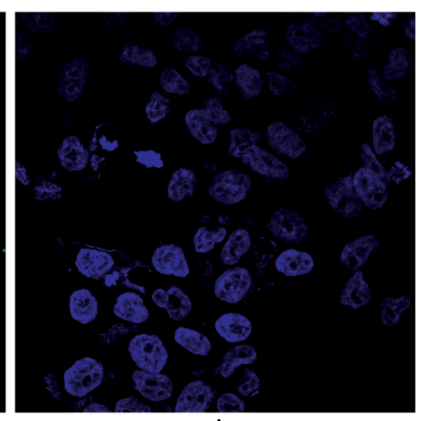

b
Fig. 8 Visualization of LNCaP cells by confocal microscopy: (a) cells treated with modified 2D-BN nanoparticles and (b) reference cells (green fluorescence - FITC, blue fluorescence - Hoechst 33258).

registered. The 2D-BN nanoparticles can enhance the scattering intensity without distorting the shape and frequency of the Raman bands. This could be due to dielectric property of 2D-BN nanoparticles which does not change the dielectric environment of the cell in contrast to metallic ones.

\section{Visualization of FITC-modified 2D-BN nanoparticles in cells}

LNCaP cells were grown on $1 \mathrm{~cm}^{2}$ coated glass beads in a 24-cup plate in an amount of $5 \times 10^{4}$ cells per cup. The following day, FITC-modified 2D-BN dispersion (final concentration in the nutrient medium was $100 \mu \mathrm{g} \mathrm{mL}^{-1}$ ) was added to the cups, and the medium was removed after $24 \mathrm{~h}$ incubation, washed with PBS, fixed with $4 \%$ paraformaldehyde, washed 3 times for $5 \mathrm{~min}$ using PBS-T (PBS with $0.2 \%$ Triton X-100), stained with a 3258 (Sigma) dye for $5 \mathrm{~min}$, washed with PBS-T, and visualized using a LSM 510 META (Zeiss) confocal microscope (Fig. 8).

Visualization of cells by confocal microscopy without dyes. Confocal images ${ }^{27-31}$ of LNCaP cells grown on different substrates (glass and gold) as well as those treated with the 2DBN nanoparticles and Dox are shown in Fig. 9-12. Luminescence confocal images were obtained by a confocal laser scanning microscope Carl Zeiss LSM-510 META with Plan-Neofluar $63 \times / 0.75$ Korr and $40 \times / 0.6$ Korr objectives. The fluorescence images were registered by using Zeiss AxioCam digital camera. The PL excitation was carried out using an ultra-violet lamp HBO 100 and blue (FSet01 wf), green (Fset10 wf), or red (Fset20 wf) filters. It is seen that the cells possess non-specific luminescence, which is enhanced when the cells are grown on the gold substrates.

Dox itself demonstrates strong luminescent properties, and therefore cells treated with Dox are well visualized. In particular, condensed chromatin of various densities in the form of light spots is clearly visible in the image of LNCaP cells treated with Dox. The chromatin condensation process could indicate necrosis of LNCaP cells provoked by Dox.

\section{Discussion}

The vibrational spectra of the studied samples showed that 2DBN nanoparticles strongly affect the structure of the cell 

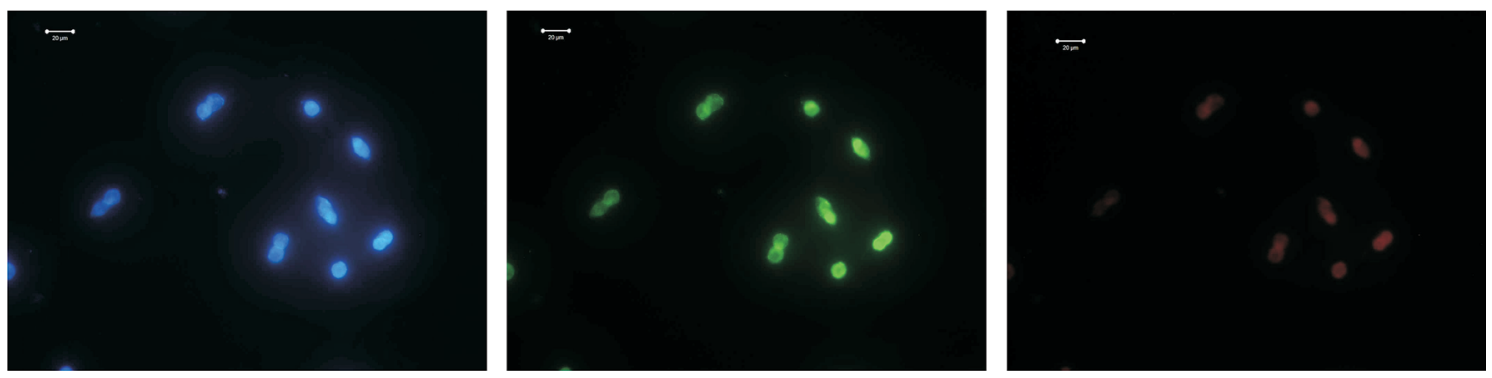

Fig. 9 Confocal microscopy images of LNCap cell treated with 2D-BN, on glass substrate.
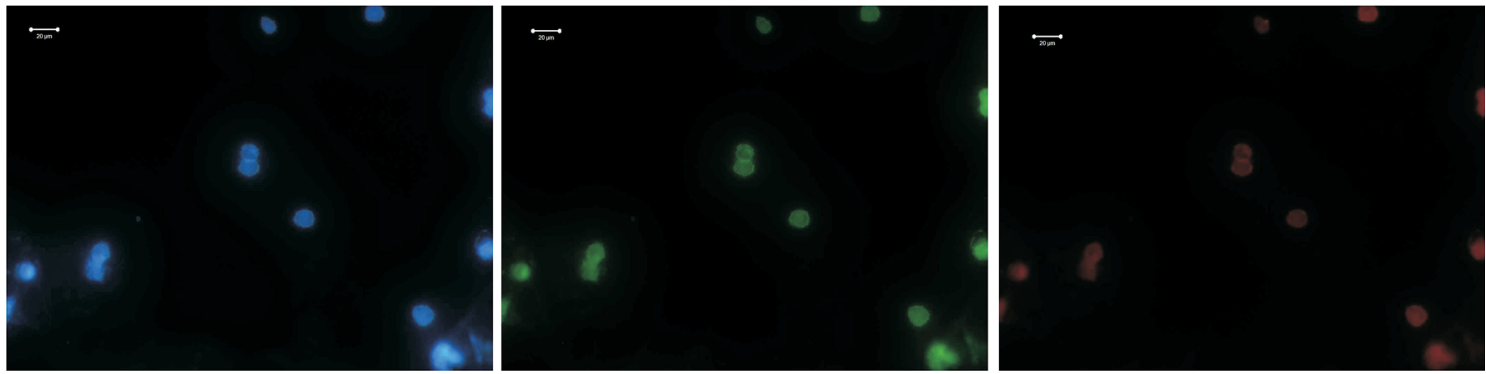

Fig. 10 Confocal microscopy images of LNCap cell treated with 2D-BN, on gold substrate.
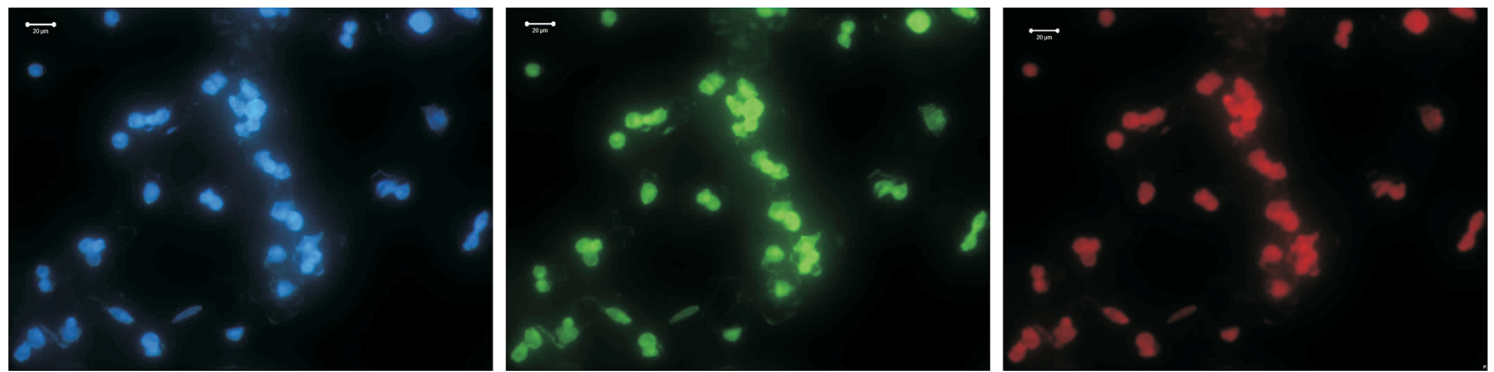

Fig. 11 Confocal microscopy images of LNCap cell treated with Dox on gold substrate.

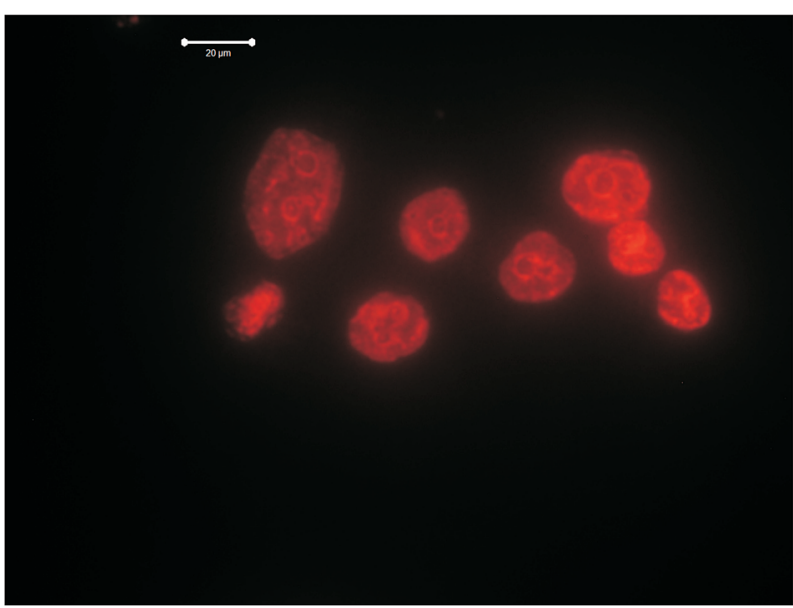

Fig. 12 Confocal microscopy images of LNCap cell treated with Dox on gold substrate. membranes incubated for 1 hour. Strong variation in $\mathrm{CH}$ stretching vibrations of the cell membrane in the FTIR spectra, a significant reorganization of the Raman spectra of the cells after treatment, the confocal microscopy images showing the presence of the nanoparticles not only on the cell membrane, but also inside the cells, all confirm the interaction of the nanoparticles with the cell membrane.

Incubation of the 2D-BN-treated cells during longer periods of time did not lead to alike changes neither in FTIR or Raman spectra. That allowed one to suggest that the nanoparticles penetrate through the cell membrane and localize in the cell cytoplasm or even near the cell nucleus. The nanoparticles which do not pass through the membrane may be deposited on its surface; however this disturbs the cell membrane only slightly.

Of course, the mechanism of the nanoparticles penetration through the cell membrane remains unclear yet. We could assume the phagocytosis mechanism, because we managed to 
register using Raman spectroscopy a strong perturbation of the cell membrane after the $2 \mathrm{D}$-BN cell treatment which we believe could be recorded for strong membrane distortion state and even at the initial state of the nanoparticle-bearing ball separation from the membrane. At this very moment, the lipids in the examined site of the membrane are strongly disordered, some of the chemical bonds are broken and new hydrogen bonds are formed, which was indicated by the increase in the intensity of the symmetric phosphate absorption bands and the redistribution of the intensity ratio between the absorption bands assigned to different phosphate groups, as well as significant enhancement of intensity of the $\mathrm{CH}$ stretching vibrations (Fig. 3).

According to FTIR spectroscopy, strong membrane perturbation occurred during $1 \mathrm{~h}$ incubation. Further cells incubation did not cause such spectral changes.

Confocal microscopy data showed that the tumour cells exhibit insignificant level of fluorescence without dyes, but luminescence and contrast of the images increase for cells being adsorbed on the nanostructured gold surfaces. Labelling of the cells with 2D-BN nanoparticles enhanced the contrast under UV excitation and using blue and green filters. When the red filter was used, the image was barely visible. The fluorescence of 2D-BN was in the range of $350 \mathrm{~nm}$ and absorption of Dox approximately coincides with the fluorescence of gold (540 $\mathrm{nm}$ ) that could cause an increase in the image contrast. The incubation of the cells resulted in a much more contrasting luminescence images in the red filter. More striking lines on the cell's image most likely referred to places of Dox localization in the cell. The image became less pronounced upon insertion of DOX and 2D-BN in the cells. We concluded that DOX and 2D-BN mutually quench fluorescence of each other in the presence of the gold nanostructured surface, and act collectively to quench the luminescent cell channels.

Based on the analysis of confocal images, we concluded that the cell fluorescence can be enhanced by using the gold surface. When the cell was incubated with DOX on the gold surface, the image obtained with the red filter was amplified. When the cell was incubated with 2D-BN, the image obtained using blue or green filter was amplified. When the cells are incubated with DOX and 2D-BN, the cell fluorescence is quenched. These evidences could indicate the DOX and 2D-BN entering into the

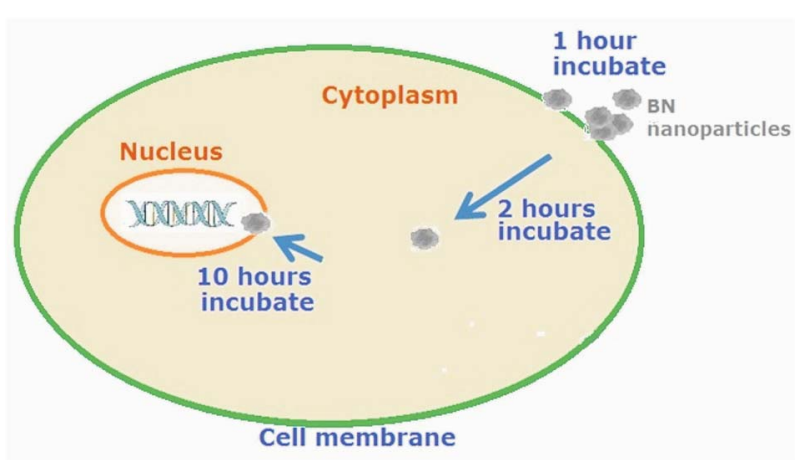

Fig. 13 Scheme of passing the nanoparticles through the cell membrane. cell. Thus, both 2D-BN interaction with the cell and 2D-BN insertion into the cell could be used for more efficient cell labelling. The most interesting result here was that 2D-BN nanoparticles exhibit protective properties against Dox action on the normal cells when administered together with the nanoparticles. Meanwhile, the Dox drug effect on the malignant cells did not change.

\section{Conclusions}

It was shown that 2D-BN nanoparticles prepared by us do not exhibit cytotoxicity in relation to normal cells. In a complex with Dox, the nanoparticles did not change the effectiveness of the drug effect on the tumour cells.

We were able to register the moment of the tumour cell membrane perturbation by the 2D-BN nanoparticles using FTIR and Raman spectroscopy. The analysis of the spectra and confocal images showed that the nanoparticles pass into the cells, localize in the cytoplasm and come near the nucleus (Fig. 13). The details of the interaction of 2D-BN nanoparticles with the tumour cell components were analysed.

2D-BN nanoparticles, along with the nanostructured golden surface, can be used to enhance the contrast of the cell images in confocal microscopy, and can serve as markers to control the penetration of drugs into the cell, both normal and tumour. Cells labelled with Dox on the gold nanostructured surface produce enhanced fluorescence images when excited by ultraviolet light with the red filter.

The cells incubated with DOX and adsorbed on the gold surface can provide information in confocal microscopy on whether the DOX interacts with the cell and what changes in the structure occur there. The features of the luminescent cell images could be used for analytical purposes.

DOX modified with 2D-BN nanoparticles has the same efficiency on malignant cells as without nanoparticles, but its protective effect on normal cells increases.

The 2D-BN nanoparticles can be used to enhance the Raman scattering intensity from the cell components.

\section{Conflicts of interest}

There are no conflicts of interest to declare.

\section{Acknowledgements}

This work has been supported by STCU 6175, NATO 5291, 690853-assymcurv-H2020-MSCA-RISE-2015/H2020-MSCA-RISE2015, NASU "Development of 2D materials for smart sensors of biomedical application” projects.

\section{Notes and references}

1 F. Alexis, E. M. Pridgen, R. Langer and O. C. Farokhzad, Handb. Exp. Pharmacol., 2010, 197, 55.

2 A. Sharma, A. K. Goyal and G. Rath, J. Drug Targeting, 2017, $15,1$. 
3 K. Hui Son, J. Hee Hong and J. Woo Lee, Int. J. Nanomed., 2016, 11, 5163.

4 S. Reich, A. C. Ferrari, R. Arenal, A. Loiseau, I. Bello and J. Robertson, Phys. Rev. B: Condens. Matter Mater. Phys., 2005, 71, 205201.

5 Md. Alim Iftekhar Rasel, T. Li, T. Dung Nguyen, S. Singh, Y. Zhou, Y. Xiao and Y. Tong Gu, J. Nanopart. Res., 2015, 17, 441.

6 G. Ciofani, V. Raffa, A. Menciassi and A. Cuschieri, Biotechnol. Bioeng., 2008, 101, 850.

7 M. Emanet, Ö. Sen, Z. Cobandede and M. Culha, Colloids Surf., B, 2015, 134, 440.

8 Q. Weng, B. Wang, X. Wang, N. Hanagata, X. Li, D. Liu, X. Wang, X. Jiang, Y. Bando and D. Golberg, ACS Nano, 2014, 8, 6123.

9 T. J. Anchordoquy, Y. Barenholz, D. Boraschi, M. Chorny, P. Decuzzi and others, ACS Nano, 2017, 11(1), 12.

10 I. V. Sukhorukova, I. Y. Zhitnyak, A. M. Kovalskii, A. T. Matveev, O. I. Lebedev, X. Li, N. A. Gloushankova, D. Golberg and D. V. Shtansky, ACS Appl. Mater. Interfaces, 2015, 7, 17217.

11 M. Dalmark, Scand. J. Clin. Lab. Invest., 1981, 41, 633.

12 G. Arancia, F. Bordi, A. Calcabrini, M. Diociaiuti and A. Molinari, Pharmacol. Res., 1995, 32, 255.

13 A. Pakdel, Y. Bando and D. Golberg, Chem. Soc. Rev., 2014, 43, 934.

14 R. V. Gorbachev, I. Riaz, R. R Nair, R. Jalil, L. Britnell, B. D. Belle, E. W. Hill, K. S. Novoselov, K. Watanabe, T. Taniguchi, A. K. Geim and P. Blake, Small, 2011, 7, 465. 15 Y. Lin and J. W. Connell, Nanoscale, 2012, 22(4), 6908.

16 O. Yu Posudievsky, O. A. Khazieieva, V. V. Cherepanov, G. I. Dovbeshko, V. G. Koshechko and V. D. Pokhodenko, RSC Adv., 2016, 6, 47112.
17 N. Sampson, H. Neuwirt, M. Puhr, H. Klocker and I. E. Eder, Endocr-Relat. Cancer, 2013, 20, R49.

18 O. Ilchenko, Y. Pilgun, T. Makhnii, R. Slipets, A. Reynt, A. Kutsyk, D. Slobodianiuk, A. Koliada, D. Krasnenkov and V. Kukharskyy, Vib. Spectrosc., 2016, 83, 180.

19 E. Goormaghtigh, J.-M. Ruysschaert and V. Raussens, Biophys. J., 2006, 90(8), 2946.

20 Z. Movasaghi, S. Rehman and Dr I. ur Rehman, Appl. Spectrosc. Rev., 2008, 43(2), 134.

21 G. I. Dovbeshko, N. Ya. Gridina, E. B. Kruglova and O. P. Pashchuk, Talanta, 2000, 53, 233.

22 O. P. Repnytska, G. I. Dovbeshko, V. P. Tryndiak, I. M. Todor and D. V. Kosenkov, Faraday Discuss., 2004, 126, 61.

23 G. Dovbeshko, V. Chegel, N. Gridina, O. Repnytska, Y. Shirshov, V. Tryndiak, I. Todor and G. Solyanik, Biospectroscopy, 2002, 67, 470.

$24 \mathrm{H}$. Arakawa, G. Dovbeshko, S. Diamantoglou and H.-A. Tajmir-Riahi, DNA Cell Biol., 2010, 29(8), 459.

25 D. R. Whelan, K. R. Bambery, L. Puskar, D. McNaughton and B. R. Wood, Analyst, 2013, 138, 3891.

26 Z. Movasaghi, S. Rehman and Dr I. U. Rehman, Appl. Spectrosc. Rev., 2007, 42(5), 493.

27 W. Han, L. Liu, T. K. Sham and Z. Liu, Nanoscale, 2012, 4, 6951.

28 S. Paddock, Confocal microscopy methods and protocols, Humana Press, Newcastle, 1998.

29 H. W. Robert, Rep. Prog. Phys., 1996, 59, 427.

30 R. Rowland and E. Nickless, Bioscene, 2000, 26(3), 1.

31 P. Verveer, M. Gemkow and T. Jovin, J. Microsc., 1999, 193, 50 . 